\title{
A model of rotated mirror/normal letter discriminations
}

\author{
Eva Kung AND JefF P. HAMM \\ University of Auckland, Auckland, New Zealand
}

\begin{abstract}
Rotated mirror/normal letter discriminations are thought to require mental rotation in order to determine the direction of facing of the stimulus. The response time (RT) function over orientation tends to be curved, rather than the linear function found for other mental rotation tasks. The present study investigated the possibility that the curved RT function is a result of a mixture of trials requiring and not requiring mental rotation. The results suggested that the frequency of mental rotation is also a linear function of stimulus orientation. Moreover, the relationship between an individual's rate of plane rotation and the mean difference in RT between mirror and normal stimuli was replicated, supporting the suggestion that mirrored stimuli are flipped after they are spun (Hamm, Johnson, \& Corballis, 2004). On the basis of the present findings, the entire RT function can be modeled by using only the mean RTs for upright and inverted stimuli.
\end{abstract}

Rotated mirror/normal letter discriminations are thought to be performed by mentally rotating the stimulus to the upright prior to making the mirror/normal judgment (Cooper \& Shepard, 1973; Corballis \& McLaren, 1984; Hamm, Johnson, \& Corballis, 2004). However, the response time (RT) function for rotated mirror/normal letter discriminations deviates from the linear function shown for other tasks thought to employ mental rotation, such as the same/different judgments of 3-D block figures (Shepard \& Metzler, 1971) or left-/right-facing judgments of common objects (Jolicœur, 1988; Murray, 1997). The RT function for mirror/normal judgments of rotated letters tends to be curved, rather than strictly linear, with the slope of the function increasing with stimulus orientation (Cooper \& Shepard, 1973; Corballis \& McLaren, 1984; Hamm et al., 2004; Heil, Rauch, \& Hennighausen, 1998). This change in the relationship between stimulus orientation and RT requires explanation, given that the strictly linear relationship is one of the signature characteristics of mental rotation (Shepard \& Metzler, 1971). Indeed, deviations from linearity have been suggested to reflect the influence of additional processes in some tasks (Jolicœur, 1990) or to reflect alternative processing strategies (Murray, 1997).

To explain the curved RT function, Cooper and Shepard (1973) originally suggested that mental rotation may be faster for more familiar stimuli and that near upright letters are more familiar than more inverted letters. This would account for the deviation from linearity during mirror/normal judgments, because the stimuli that are rotated more are rotated more slowly. However, rotated common objects would likewise be less familiar and left-/ right-facing judgments do not show this change in men- tal rotation rates (Murray, 1997), which works against the idea that mental rotation rates may vary with respect to familiarity. Cooper and Shepard also suggested that during mirror/normal letter discriminations, mental rotation may not be employed on every trial when the stimulus is near the upright. This would suggest that the mean RT comprises a mixture of rotation and nonrotation processes, with more rotated stimuli requiring a greater proportion of trials that employ mental rotation, which results in the curved RT function.

Another aspect of the RT pattern found for this task is that the RTs for mirror judgments tend to be longer than the RTs for normal ones. This was originally suggested as possibly reflecting participants' tendency to prepare a normal response (Cooper \& Shepard, 1973), which would result in a general RT benefit for normal responses. An alternative explanation is that mirror stimuli may be fully normalized, meaning that they are flipped after they are plane rotated (Hamm et al., 2004). This alternative is, in part, based on analysis of event-related potentials (ERPs), where the upright mirror-normal difference wave revealed a highly similar difference component as the ERP marker for plane rotation (Hamm et al., 2004). In addition, it was shown that there was a correlation between an individual's rate of plane rotation and the magnitude of the mirror-normal difference, with slow plane rotators showing larger mirror-normal differences (Hamm et al., 2004). This relationship is consistent with the interpretation of the ERP data that suggests that the mirror-normal difference reflects the additional mental rotation required to flip the stimulus after the plane rotation has been completed.

If the deviation from pure linearity that is often found for rotated mirror/normal judgments of alphanumeric stimuli

J. P. Hamm, j.hamm@auckland.ac.nz 
is due to some trials near the upright not requiring mental rotation, it is of interest to determine the relationship between stimulus orientation and this nonrotation route that appears able to perform mirror/normal judgments. To investigate what will be referred to as the mixture hypothesis, the following three assumptions will be made. First, mental rotation is never required for upright stimuli, apart from the proposed "flip" if the stimulus is mirrored (Hamm et al., 2004). Second, mental rotation is always employed for inverted stimuli. This second assumption is simply stating the status quo, that rotated mirror/normal letter discriminations require mental rotation in order to determine the facing of the character. Although this assumption is not stated directly in the literature, it is implied by the reporting of the effect in terms of rates of rotation, because assuming a mixture would then lead to the conclusion that the rate cannot be recovered without removing the nonrotation trials. The third assumption is that on trials in which mental rotation is not employed despite the stimulus being presented in an orientation other than upright, the mean RT will be equal to the mean RT to upright stimuli. This assumption is based on the fact that tasks involving decisions about rotated stimuli that are not thought to involve mental rotation, such as letter/digit classification, generally show flat RT functions (Corballis \& Nagourney, 1978; Corballis, Zbrodoff, Shetzer, \& Butler, 1978; Heil, Bajric, Rosler, \& Hennighausen, 1996; Milivojevic, Hamm, \& Corballis, 2009), and so a similarly flat function is proposed.

From these assumptions, an individual's rate of rotation is best estimated by the difference in RTs between inverted and upright stimuli, since other orientations do not purely reflect mental rotation. This allows one to estimate the mean RT for trials on which mental rotation is employed at each orientation by linear interpolation (Shepard \& Metzler, 1971). Using the observed RT for upright stimuli as the estimate for the nonrotated trials and the estimated RTs due to mental rotation, the proposed proportion of trials that employ mental rotation at each orientation can be recovered. For example, if a participant has a mean RT of $400 \mathrm{msec}$ for upright stimuli and $760 \mathrm{msec}$ for inverted stimuli, this translates into a rate of rotation of $2 \mathrm{msec} / \mathrm{deg}[(760-400) / 180]$. If we observed a mean RT of $409 \mathrm{msec}$ for stimuli rotated $30^{\circ}$, this is less than the expected time of $460(400+2 * 30)$. By assuming that $409=\left(p_{\mathrm{MR}} * 460\right)+\left(1-p_{\mathrm{MR}}\right) * 400$, where $p_{\mathrm{MR}}$ is the proportion of trials on which mental rotation is employed, we can then simply solve for this proportion: $p_{\mathrm{MR}}=.15$. This would suggest that only $15 \%$ of the trials required mental rotation. ${ }^{1}$

Therefore, the purpose of the present experiment is to investigate the possibility of explaining the curved RT function produced during the rotated mirror/normal letter discrimination task as resulting from the mixture of trials on which mental rotation is and is not employed. The mixture hypothesis will be used as the basis for estimating the proportions for each of these types of trials at each orientation to determine at what degree of rotation from the upright mental rotation appears to be consistently employed and to determine whether there is any systematic relationship between orientation and the mixture proportions.

\section{METHOD}

\section{Participants}

Twenty-six participants voluntarily took part in this study. All had normal or corrected-to-normal vision. Although English was not required as a first language, all the participants were fluent readers of English text. Data from 2 participants were excluded from the analysis, due to failure to meet the performance criterion outlined in the Procedure section below. All the results presented are based on the remaining 24 participants, whose ages ranged from 18 to 27 years $(M=20.8, S D=2.4 ; 12$ male). Twenty-one participants were assessed as right-handed and 3 as ambidextrous (but with a positive score) on the Edinburgh Handedness Inventory (Oldfield, 1971). All procedures were approved by the University of Auckland Human Participants Ethics Committee.

\section{Stimuli}

The six uppercase alphabetic characters (F, G, J, L, P, and R) were used as stimuli and are shown in Figure 1 in their normal version. A chinrest was used to maintain the participants' viewing distance at $57 \mathrm{~cm}$ from their eyes to the center of the screen. The stimuli shown in Figure 1 were scaled such that their vertical axis spanned $4.8^{\circ}$ of visual angle (when upright). Each letter was presented in both normal and mirror-reversed form 24 times at every orientation ranging from $0^{\circ}$ to $330^{\circ}$, in $30^{\circ}$ steps clockwise per block of trials. The stimuli were presented in a random order and on a color computer monitor with a screen resolution of $800 \times 600$ pixels. The letters were presented on a gray background. Response timing began by first synchronization with the screen refresh cycle, as suggested by Heathcote (1988), and further synchronization with the uppermost pixel of the stimulus, using the timing routines described by Hamm (2001).

\section{Procedure}

Four blocks of 576 trials were conducted in which response hand (left or right) and response finger assignment (normal to index and mirror to middle or mirror to index and normal to middle) were counterbalanced over blocks. This resulted in each participant's performing 2,304 trials. There were four self-timed breaks within each block. Each block required the participant to determine whether the letters were normal or mirror reversed. The participants indicated their response by pressing a " 1 " or a " 2 " on a numeric keypad, with the assignment of normal and mirror to the keys depending on the particular block conditions. Conditions 1 and 2 required the participants to respond with their left hand, with the keys assigned $1=$ normal and $2=$ mirror for Condition 1 . The reverse assignment was used for Condition 2. Conditions 3 and 4 required the participants to respond with their right hand, with Condition 3 assigning the keys as $1=$ normal and $2=$ mirror and the reverse assignment for Condition 4. Conditions were counterbalanced using a Latin square.

The entire experiment required approximately $1 \mathrm{~h} 40 \mathrm{~min}$ to complete. The participants had the option of completing the experiment in one session or in two sessions of two blocks each. The range of completion times was from same day (11 participants) up to 1 week (2 participants).
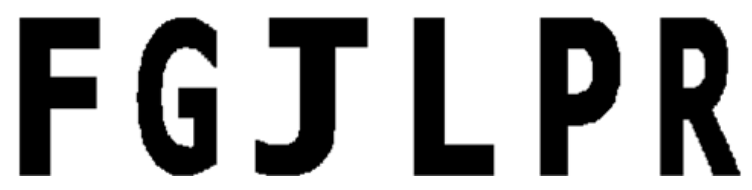

Figure 1. Normal version of letters used as stimuli in the present study. 
The inclusion criterion for the analysis required the participant to reach a performance level of at least $85 \%$ correct overall, with no individual cell of the design (hand $\times$ finger $\times$ orientation $\times$ character facing) being less than $60 \%$ correct.

\section{RESULTS}

Note that in all the figures, the data presented at $360^{\circ}$ are duplicated from the $0^{\circ}$ data and are included only to aid the visual assessment of symmetry, but the actual data are entered into the analysis only once.

\section{Mean RT}

Mean RTs were calculated for correct responses only, and outliers were removed using a sliding criterion to avoid bias (Van Selst \& Jolicœur, 1994). In a preliminary analysis, the mean RTs were analyzed in a five-way mixed factor ANOVA, with gender (2), character facing (2), hand (2), finger (2), and orientation (12) as factors. Planned contrasts were used to test for symmetry in the orientation effect by comparing $30^{\circ}$ with $330^{\circ}, 60^{\circ}$ with $300^{\circ}, 90^{\circ}$ with $270^{\circ}, 120^{\circ}$ with $240^{\circ}$, and $150^{\circ}$ with $210^{\circ}$ of clockwise rotation, with an additional six contrasts employed to perform a complete trend analysis and test for a linear through sixth-order trend component between $0^{\circ}$ and $180^{\circ}$ and combining clockwise with counterclockwise orientations. This analysis indicated a main effect of response finger, with the index finger $(777 \mathrm{msec})$ producing shorter RTs than did the middle finger [793 msec; $F(1,22)=7.43, p<.05]$. However, this effect of finger did not interact with any other factor. There was no effect of hand or gender, and no interactions involved these factors, apart from an interaction between gender and the fifth-order trend component of the orientation factor $[F(1,22)=5.66, p<.05]$. Since this higher order trend component currently has no known theoretical interest, it will not be discussed further.

On the basis of the preliminary analysis, the mean RT data were collapsed and analyzed in a two-way withinsubjects ANOVA, with character facing (2) and orientation (12) as factors. The same set of contrasts was used to examine the orientation factor. The mean RTs may be seen in Figure 2A.

This analysis resulted in a significant main effect for character facing $[F(1,23)=84.55, p<.05]$, with normal letters $(716 \mathrm{msec})$ responded to more quickly than mirror letters $(854 \mathrm{msec})$. There was a significant main effect for orientation $[F(11,253)=99.08, p<.05]$, for which only the contrasts testing for linear $[F(1,23)=198.50$, $p<.05]$, quadratic $[F(1,23)=59.12, p<.05]$, and cubic $[F(1,23)=11.59, p<.05]$ trends reached significance. The interaction between character facing and orientation reached significance $[F(11,253)=2.32, p<.05]$. The contrasts indicated a significant interaction between character facing and the test for symmetry between $30^{\circ}$ and $300^{\circ}[F(1,23)=4.58, p<.05]$ and an interaction in the linear trends $[F(1,23)=7.92, p<.05]$.

If the difference between the RTs to mirror and normal letters is due to an additional flip after, or near completion of, the plane rotation (Hamm et al., 2004), there should be a relationship between participants' mental rotation rates and the magnitudes of their mirror-normal letter difference. To test this, each participant's mental rotation rate was estimated as the mean difference between upright and inverted stimuli, collapsed over mirror and normal, divided by 180 to give a millisecond/degree measure of rate $\left(\mathrm{MR}_{\text {rate }}\right)$. This was used as the predictor of the participant's mean mirror-normal difference $\left(\mathrm{MN}_{\text {diff }}\right)$ collapsed over orientation. A significant positive relationship was found $\left[r^{2}=.34 ; F(1,22)=11.42, p<.05\right.$; the regression equation that results is $\left.\mathrm{MN}_{\text {diff }}=52.39 \mathrm{MR}_{\text {rate }}+20.37\right]$, indicating that the participants with slower rates of rotation also tended to have a bigger mirror-normal difference. Neither the participants' mental rotation rate nor their mirror-normal difference was significantly correlated to their mean RTs for upright normal letters (both $p s>.10$ ), which was used as an index of general processing efficiency (Hamm et al., 2004). This replicates the findings of a similar relationship found in the behavioral data reported in a previous EEG study (Hamm et al., 2004).

A similar two-way ANOVA was performed on response accuracies, which are shown in Figure 2B. Nothing was found to indicate that any of the findings above were due to speed-accuracy trade-offs. The main effect for character facing did not reach significance $[F(1,23)=0.65, p>$ .05]. There was a significant main effect for orientation $[F(11,253)=19.73, p<.05]$, for which only the contrasts comparing $120^{\circ}$ with $240^{\circ}[F(1,23)=4.71, p<.05]$ and $150^{\circ}$ with $210^{\circ}[F(1,23)=6.68, p<.05]$ and the linear $[F(1,23)=56.36, p<.05]$ and quadratic $[F(1,23)=$ $19.73, p<.05]$ trends reached significance. There was a significant interaction between character facing and orientation $[F(11,253)=3.29, p<.05]$, for which only the contrasts testing for the interaction of character facing and the linear $[F(1,23)=6.65, p<.05]$ and quadratic $[F(1,23)=6.26, p<.05]$ trends reached significance.

\section{Proportions of Trials on Which Mental Rotation Was and Was Not Employed}

The estimated proportions of trials on which mental rotation was thought to have been employed were calculated, with the assumption that the proportion would be 0 for upright stimuli and 1 for inverted stimuli (100\% mental rotation). Because of the interaction found between mirror and normal stimuli and the linear trend for orientation, the rates of rotation were calculated separately for mirror and normal stimuli. Rates of rotation were estimated from the corresponding difference in RTs between upright and inverted stimuli, rather than from slopes of regression lines, to avoid any relationship between the estimate of the rate of rotation and the data under examination. Because proportions for $0^{\circ}$ and $180^{\circ}$ were 0 and 1 by definition, only the values at the intermediate orientations were free to vary. The proportions were estimated for each participant for statistical analysis.

A three-way within-subjects ANOVA was performed on the proportion of rotation values, with character facing (2), direction from upright (2; clockwise vs. counterclockwise), and orientation $\left(5 ; 30^{\circ}-150^{\circ}\right)$ as factors. Planned contrasts were used to perform a complete trend 


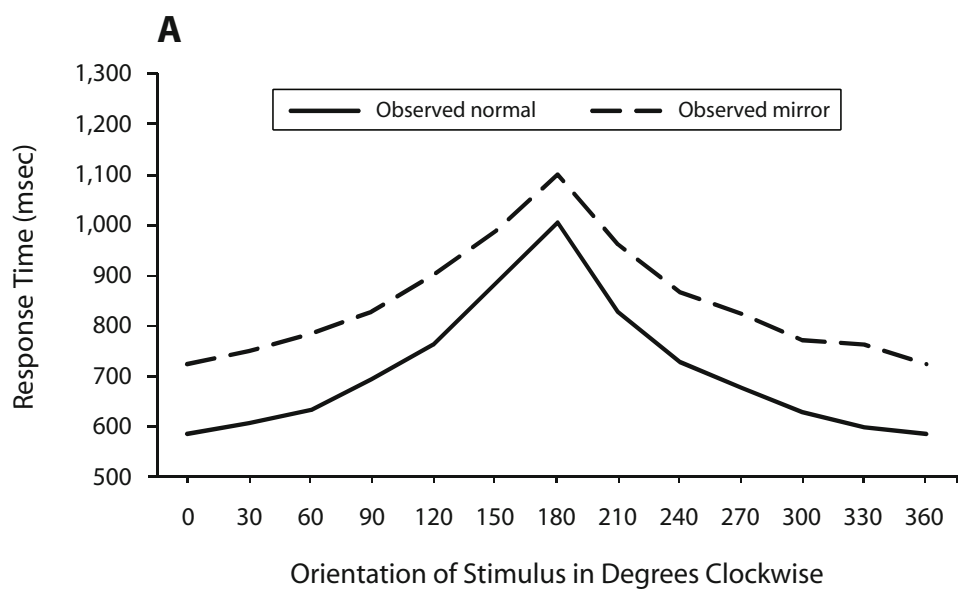

B

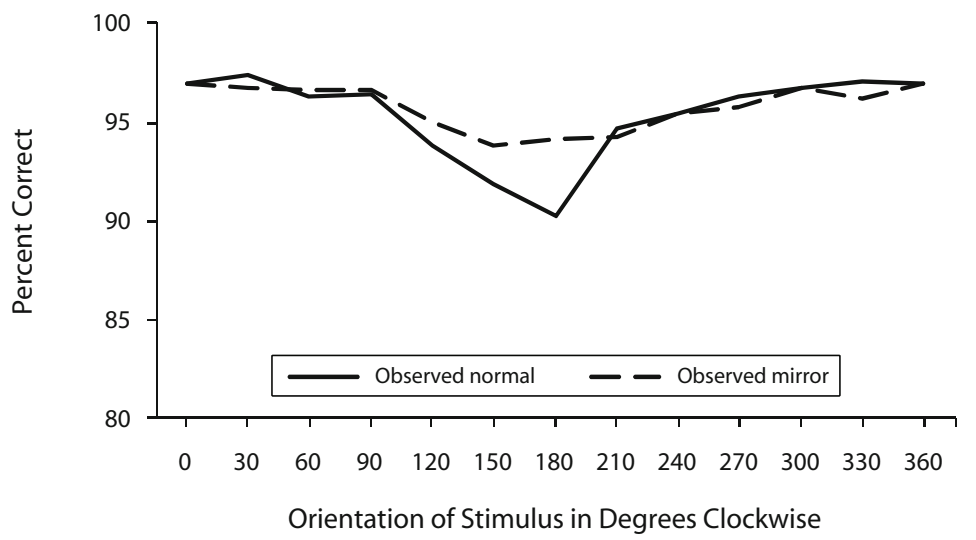

Figure 2. The mean observed response times (A) and percent correct (B) as a function of stimulus orientation in response to mirrored (dashed lines) and normal (solid lines) stimuli.

analysis and to test for the linear through fourth-order trend components. The only significant result was a main effect for orientation $[F(4,92)=20.37, p<.05]$, for which only the contrast testing the linear trend reached significance $[F(1,23)=30.57, p<.05]$. Examinations of the proportions of mental rotation trials, shown in Figure 3, suggested that mental rotation may have been employed in proportion to the degree to which the stimulus was inverted. This relationship converts orientation to proportional inversion as stimulus angle divided by 180 , which is represented in Figure 3 by the dashed line and is reflected in the values of the $y$-axis. Proportions calculated for each of the individual letter stimuli did not reveal any systematic differences between the characters, with the presented proportions representative across the stimulus set.

\section{Estimating Rotated Mirror and Normal RTs From Mean Upright and Inverted RTs}

The proportion of trials on which mental rotation $\left(p_{\mathrm{MR}}\right)$ was employed increases linearly as a function of the stim- ulus's orientation away from upright. This would suggest that, under the present experimental conditions at least, $p_{\mathrm{MR}}=\theta / 180$, where $\theta$ is the orientation of the stimulus in degrees from the upright. Therefore, the mean RT can be modeled as a simple weighted average between the nonrotation trials, with a mean equal to that of the upright condition, and the rotation trials, with a mean RT estimated on the basis of the assumption of a linear increase in the RT between $0^{\circ}$ and $180^{\circ}$. In other words, $\mathrm{RT}_{\text {(predicted) }}=$ $p_{\mathrm{MR}}\left(\mathrm{RT}_{(\mathrm{MR})}\right)+\left(1-p_{\mathrm{MR}}\right)\left(\mathrm{RT}_{(\sim \mathrm{MR})}\right)$. Where $\mathrm{RT}_{(\text {predicted })}$ is the expected mean RT based on the modeled mixture, $p_{\mathrm{MR}}$ is the proportions of trials on which mental rotation is thought to have been employed $(\theta / 180), \mathrm{RT}_{(\sim \mathrm{MR})}$ is the $\mathrm{RT}$ when the stimulus is at $0^{\circ}$ using separate values for the mirror and normal conditions, and $\mathrm{RT}_{(\mathrm{MR})}$ is the expected RT if mental rotation was used on every trial, again specific to mirror and normal conditions in order to capture the current interaction. These predicted RTs for mental rotation are based on the assumption that mental rotation does not behave differently when letters are employed 


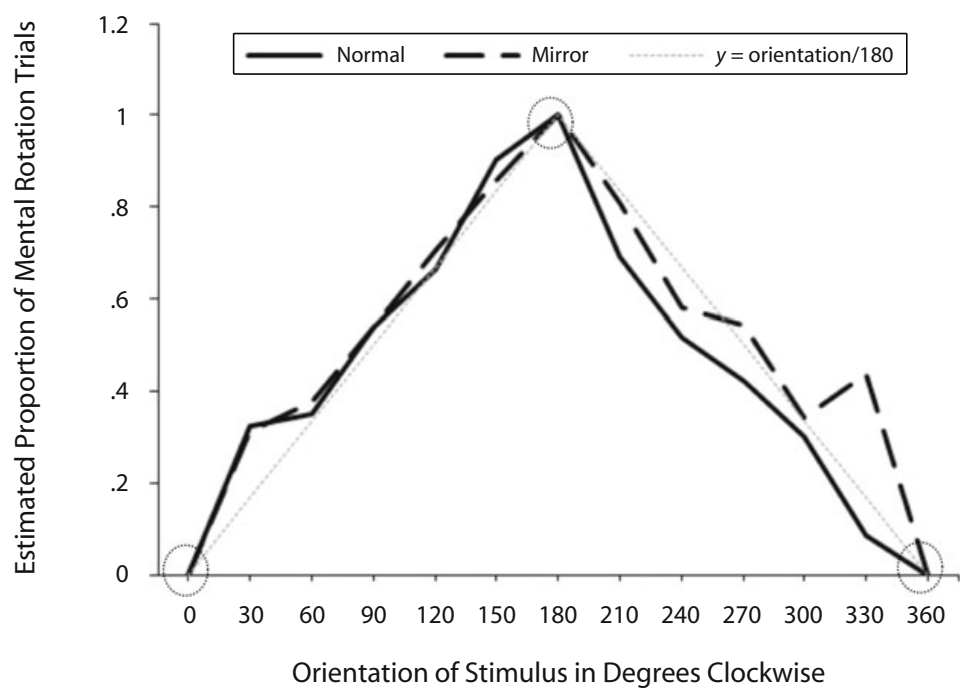

Figure 3. The proportions of mental rotation trials, with $y=$ orientation from upright/180, representing the linear increase of proportions of trials on which mental rotation was employed as a function of letter orientation in response to mirrored and normal letters. The values of 0 and 1 at $0^{\circ}$ and $180^{\circ}$ (circled) were fixed by the assumptions of the model, with the remaining values free to vary.

as stimuli than when other stimulus categories are used, meaning that it should produce a linear increase in RT. These predicted mixture values were calculated for every participant and then averaged and compared with the observed data. Figure 4A shows the model curves, with the actual data points plotted for comparison.

The interaction between facing and orientation has typically not been shown in the literature (Cooper \& Shepard, 1973; Corballis \& McLaren, 1984; Hamm et al., 2004). Therefore, rather than fit the data on the basis of separate rates of rotation for mirror and normal stimuli as above, a common rate can be used by collapsing over mirror and normal and using the difference between the RTs observed at $180^{\circ}$ and $0^{\circ}$. In addition, a common baseline RT $\left(\mathrm{RT}_{(\sim \mathrm{MR})}\right)$ can be estimated from the average RT at $0^{\circ}$. In order to recover the separation between mirror and normal RTs, the resulting curve is then shifted up or down. The magnitude of the shift is obtained by estimating the mirror normal difference $\left(\mathrm{MN}_{\text {diff }}\right)$ on the basis of an individual's rate of rotation $\left(\mathrm{MR}_{\text {rate }}\right)$, using the regression equation obtained earlier: $\mathrm{MN}_{\text {diff }}=52.39 \mathrm{MR}_{\text {rate }}+20.37$. Figure $4 \mathrm{~B}$ shows the average predicted curves after using a participant's mean RT at $180^{\circ}$ and $0^{\circ}$ collapsed over mirror and normal conditions to provide a single rate of rotation to calculate the RTs for mental rotation trials and to predict their mirror-normal difference. The error bars represent the $95 \%$ confidence intervals based on the variability of the model predictions. ${ }^{2}$

As a measure of fit, the sum of squared deviations about the participant's grand mean was compared with the sum of squared deviations about the noninteraction model predicted values (plots shown in the Appendix). The average reduction in the sum of squared deviations was $81 \%$. The range of reductions was between 59\% (Participant 13) and $92 \%$ (Participants 7, 19, and 21). As a comparison, a purely linear version of the model was evaluated by setting $p_{\mathrm{MR}}=1$ for all orientations, rather than $p_{\mathrm{MR}}=\theta / 180$. This resulted in only a $51 \%$ reduction in the variability from the mean, with 1 participant (Participant 12) showing this to be an even poorer fit than the mean alone. This analysis suggests that the model extracted from the grand average data, even though it does not model the interaction, provides good estimates for individual participant performance. Interestingly, 4 participants $(2,8,10$, and 20) did show better fits for the purely linear model than for the proposed mixture, suggesting the possibility of individual differences with respect to the process that allows for these decisions to be made without resorting to mental rotation (see Table A1 in the Appendix for a complete listing of fits for both models).

\section{DISCUSSION}

As is typical for rotated mirror/normal letter discriminations, this study showed that the RT was consistently shorter for normal letters than for mirror-reversed letters (Cooper \& Shepard, 1973). The increase in RTs as angular displacement of letters from the upright increased produced a concave function, which is also consistent with prior reports (e.g., Cooper \& Shepard, 1973; Corballis \& McMaster, 1996; Hamm et al., 2004; Heil et al., 1998). By starting with the assumption that mental rotation should produce an RT function that is purely linear as a function of stimulus orientation (Shepard \& Metzler, 1971) but that the response may be determined on some proportion of trials without resorting to mental rotation, it was possible 


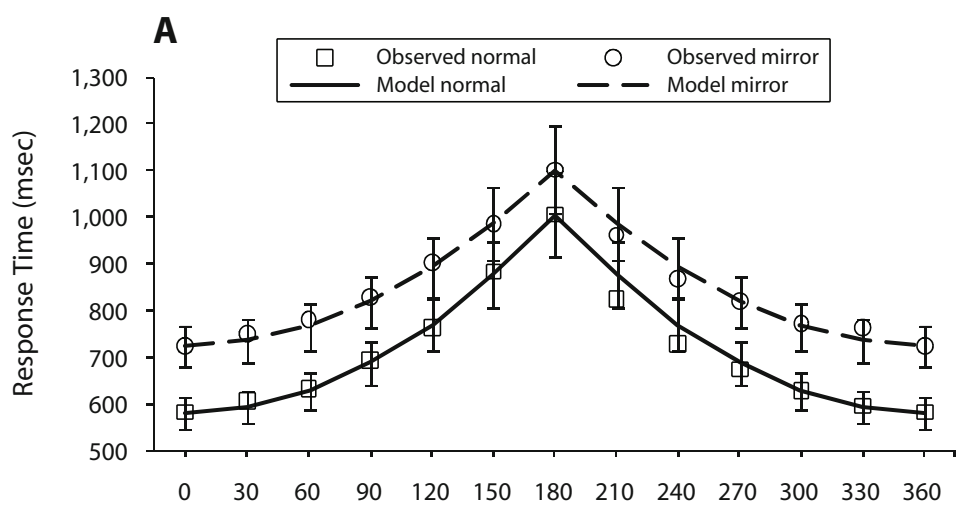

Orientation of Stimulus in Degrees Clockwise

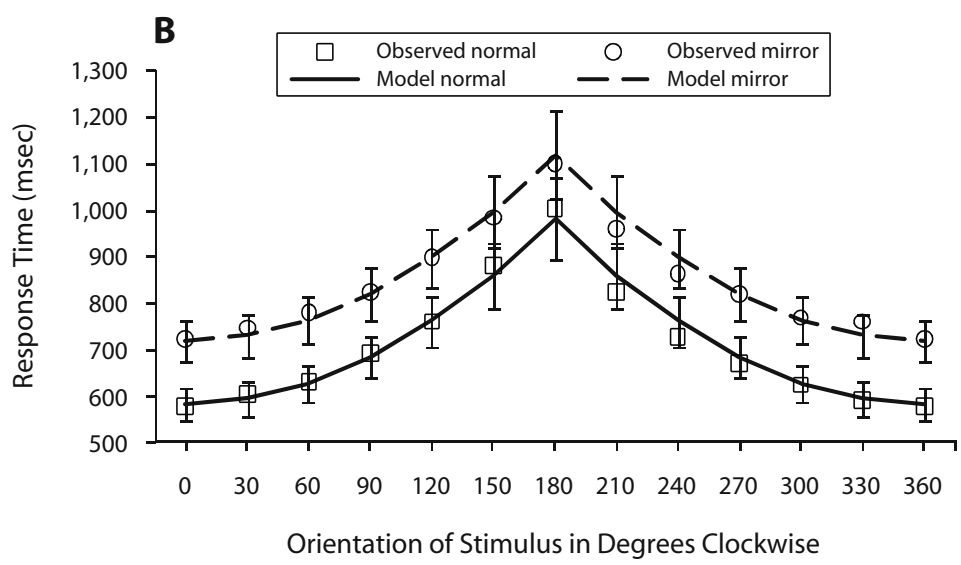

Figure 4. The mean observed and predicted response times as a function of stimulus orientation in response to mirrored and normal stimuli. Error bars about the modeled data represent $95 \%$ confidence intervals based on the variability of the modeled values for each participant at each orientation. Note that the values at $0^{\circ}$ and $180^{\circ}$ are forced in the calculations in panel $\mathrm{A}$, since this models the interaction between character version and facing, whereas in panel $B$, all data points are free to vary and the interaction between character facing and orientation is not modeled and the mirrornormal differences were estimated on the basis of a participant's individual mental rotation rate.

to estimate the proportion of trials on which mental rotation might have been employed. It was expected that this estimation procedure would indicate at what orientation mental rotation was consistently employed. However, it appeared that mental rotation was not consistently used, except perhaps for inverted stimuli. It should be pointed out that the latter point was not tested in this investigation but was one of the underlying starting assumptions. The relationship suggested that in the rotated mirror/normal letter task, the curvilinear RT function may arise due to the combination of a linear increase in RT for trials on which mental rotation is employed and a linear increase in the proportion of trials that require mental rotation.

In addition, the relationship between an individual's rate of plane rotation was correlated with the magnitude of their mirror-normal RT difference. This replicates pre- vious findings and suggests that the mirror-normal difference may reflect a flipping of the stimulus to fully normalize it (Hamm et al., 2004). With images of common objects, it appears that a $180^{\circ}$ flip may be faster than a $180^{\circ}$ spin (Murray, 1997).

On the basis of these findings, an individual's entire RT function can be modeled from two data points: their mean RTs for upright and inverted stimuli collapsed over mirror and normal trials. From these two data points, one can calculate a rate of rotation as being $\left(\mathrm{Rt}_{180}-\mathrm{Rt}_{0}\right) / 180$ and can use $\mathrm{Rt}_{0}$ as a baseline value. The orientation curve is then calculated by $\mathrm{Rt}_{\theta}=\theta / 180 *\left[\mathrm{Rt}_{0}+\theta *\left(\mathrm{Rt}_{180}-\right.\right.$ $\left.\left.\mathrm{Rt}_{0}\right) / 180\right]+(1-\theta / 180) * \mathrm{Rt}_{0}$, where $\theta$ indicates the orientation of the stimulus in degrees away from upright $\left(0^{\circ}-\right.$ $\left.180^{\circ}\right), \mathrm{Rt}_{\theta}$ is the predicted RT at the given orientation, and $\mathrm{Rt}_{0}$ and $\mathrm{Rt}_{180}$ are the mean $\mathrm{RTs}$ at $0^{\circ}$ and $180^{\circ}$, respec- 
tively. Therefore, the term $\left[\mathrm{Rt}_{0}+\theta *\left(\mathrm{Rt}_{180}-\mathrm{Rt}_{0}\right) / 180\right]$ simply calculates the predicted RT based on mental rotation manifesting as a linear increase in RTs between $0^{\circ}$ and $180^{\circ}$. The term $\theta / 180$ indicates the proportion of the trials on which mental rotation is thought to occur. This proportion also increases as a linear function of stimulus orientation (see Figure 3). The term $(1-\theta / 180)$ reflects the remaining proportion of trials on which the discrimination is made without mental rotation. These nonrotation trials are assumed to have a mean RT similar to that of the upright stimuli.

Because the orientation curve above is calculated using RTs collapsed over mirror and normal conditions, it does not take into account the mirror/normal effect. Although the present study reports a significant interaction between orientation and character facing, this interaction has rarely been replicated in the literature. However, when such interactions have been reported, it appears to have taken the form of reduced effects of orientation for mirrored stimuli (Duncombe, Bradshaw, Iansek, \& Phillips, 1994). If the mirrornormal difference reflects an out-of-plane flip (Hamm et al., 2004), such a flip may cascade (McClelland, 1979) with the plane rotation, which would reduce the mirrornormal difference at the more extreme orientations. Until it becomes clear under what circumstances this interaction occurs, one can model rotated mirror/normal letter discrimination under the assumption of no orientation $\times$ character facing interaction. To model the situation in which there is no interaction, the orientation RT function above can then be shifted up and down by a constant amount. The amount of the shift is one half of the predicted mirror-normal difference, which is calculated as $\mathrm{MN}_{\text {diff }}=52.39 *\left[\left(\mathrm{Rt}_{180}-\right.\right.$ $\left.\left.\left.\mathrm{Rt}_{0}\right) / 180\right)\right]+20.37$. Therefore, the RT curves for normal trials are modeled by the equation $\mathrm{Rt}_{\theta}=\left\{\theta / 180 *\left[\mathrm{Rt}_{0}+\right.\right.$ $\left.\left.\theta *\left(\mathrm{Rt}_{180}-\mathrm{Rt}_{0}\right) / 180\right]\right\}+\left[(1-\theta / 180) * \mathrm{Rt}_{0}\right]-\{52.39 *$ $\left.\left[\left(\mathrm{Rt}_{180}-\mathrm{Rt}_{0}\right) / 180\right]+20.37\right\} / 2$, whereas the $\mathrm{RT}$ curves for mirrored trials are modeled by the equation $\mathrm{Rt}_{\theta}=\{\theta / 180 *$ $\left.\left[\mathrm{Rt}_{0}+\theta *\left(\mathrm{Rt}_{180}-\mathrm{Rt}_{0}\right) / 180\right]\right\}+\left[(1-\theta / 180) * \mathrm{Rt}_{0}\right]+$ $\left\{52.39 *\left[\left(\mathrm{Rt}_{180}-\mathrm{Rt}_{0}\right) / 180\right]+20.37\right\} / 2$.

This noninteraction model was used to fit curves to each of the individual participants' data, and these fits can be seen in the Appendix. To fit the interaction, different rates of rotation can be used in the modeling of RTs for the mental rotation trials by using $\theta *\left(\mathrm{Rt}_{180 \mathrm{~N}}-\mathrm{Rt}_{0 \mathrm{~N}}\right) / 180$ and $\theta *\left(\mathrm{Rt}_{180 \mathrm{M}}-\mathrm{Rt}_{0 \mathrm{M}}\right) / 180$ for normal and mirror trials, respectively. Note that the RTs collapsed across mirror and normal trials should still be used when estimating the mirror-normal difference. Interestingly, the 4 participants who were best fit by a linear model also show little evidence for the interaction between orientation and character facing shown by the remaining 20 participants. This hints at the possibility that those who consistently use mental rotation to correct the plane rotation may also tend to consistently flip (Hamm et al., 2004).

In order to examine how well this model fits group data other than those from which it was derived, the RT data from Cooper and Shepard's (1973) Figure 1, condition N (no advance warning), were extracted and the uprightinverted difference was used to estimate the RT function expected. The predicted curve and the observed data (only the normal data are available from their figure) can be seen in Figure 5.

To summarize, the present results and analyses suggest that the impact of mental rotation on response latencies is linear during the mirror/normal letter discrimination task, despite the fact that the mean RTs show a curvilinear function. The deviation from linearity can be explained by a linear increase in the proportion of trials that require mental rotation in order to determine the correct response. In addition, the relationship between an individual's rate of plane rotation is related to the magnitude of the mirrornormal difference, replicating the behavioral findings reported in a previous EEG study (Hamm et al., 2004).

The following question remains, however: What is it about the mirror/normal letter task that allows for the response to be determined without resorting to mental rotation? The proposed model is based on the premise that mental rotation acts with the same properties on letters as it does on other types of stimuli, in that the transformation is proposed to occur at a constant speed regardless of the orientation of the stimulus. What is it about alphanumeric stimuli that allows for the nonrotation process to provide

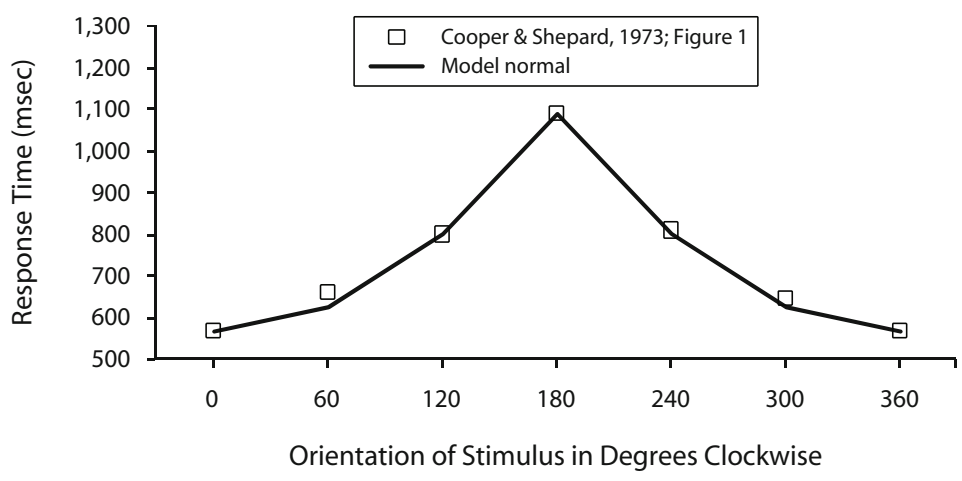

Figure 5. Cooper and Shepard's (1973) mean observed and predicted response times derived from the model employed in the present study, as a function of stimulus orientation in response to mirrored and normal stimuli. 
the correct response? One possibility may have to do with the ease of determining the polarity of the horizontal and vertical axes.

Corballis and McLaren (1984) required participants to make discriminations between vertically symmetric (i.e., $\mathrm{b}$ or $\mathrm{d}$ ? $\mathrm{p}$ or $\mathrm{q}$ ?) or horizontally symmetric (i.e., b or $\mathrm{p}$ ? $\mathrm{d}$ or q?) pairs. In one of their reported experiments (E3), a set of nonverbal (nonletter) stimuli with these characteristics were also employed. The nonverbal stimuli were very similar to the letters (a vertical rectangle with a dot beside it, rather than a line with a circle attached to the side). The RT data were extracted from their Figures 1 and 3 and were collapsed between the verbal and nonverbal stimulus conditions, and then the averaged data were used to determine the proportion of trials on which it was estimated that mental rotation was employed (see Figure 6). It appears that when the discrimination involves differentiating between horizontally symmetric stimuli (b vs. $p$ or $d$ vs. q), mental rotation is employed consistently. However, when the discrimination involves vertically symmetric stimuli, as in mirror/normal letter discriminations, mental rotation appears to be employed less frequently as a function of the stimulus orientation. When stimulus pairs are horizontally symmetric, such as $p$ versus $b$ (or $d$ vs. q), the polarity of the top-bottom axis cannot be readily determined, whereas $a b$ versus $d$ or a $p$ versus $q$ discrimination allows easy determination of the polarity of the top-bottom axis. Therefore, it may be that whatever process is employed that allows for mirror/normal discriminations to be made without mental rotation requires rapid determination of the location and the polarity of the vertical axis of the stimulus.

In a mirror/normal letter discrimination task, normal and mirrored versions of the same letter are always vertically symmetric pairs. In addition, each letter is easily identified without use of mental rotation (Heil et al., 1996; Milivojevic et al., 2009); therefore, one could determine the orientation of the stimulus prior to mental rotation. In fact,

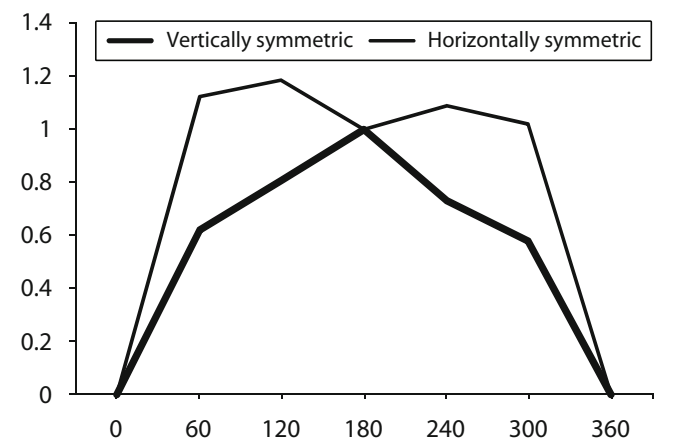

Orientation of Stimulus in Degrees Clockwise

Figure 6. Proportions of trials on which it was estimated that mental rotation was employed, from data extracted from Corballis and McLaren's (1984) study. knowing the current orientation and the normal upright are thought to be necessary priors in order to mentally rotate a stimulus through the shortest angular distance (Corballis, 1988). Knowing the current orientation suggests that the top-bottom axis has been determined and polarized into top and bottom. Locating and polarizing the horizontal axis would likewise be sufficient to determine the rotational transformation (Corballis \& McLaren, 1984), although having only this information appears to negate the use of the proposed nonrotational process.

In order to facilitate future investigations, the following is proposed as a theoretical starting point with respect to the nonrotation process that is suggested to be involved. Discrimination between mirror and normal versions of a letter requires determining whether features of the letter occur with the correct polarity along the horizontal axis of the letter. Using the letter $\mathrm{R}$ as an example, if the curve and diagonal leg are determined to be toward the right horizontal pole, the stimulus is determined to be normal. Mental rotation has been proposed to be required in order to align an image of the stimulus with a viewer-centered coordinate system that defines left and right. However, if it were possible to determine horizontal polarity without performing the imagined rotation, mental rotation would become unnecessary, and the proposed flat RT function of the nonrotation process would be the result. A nonrotation process that allows for this horizontal axis polarization should also become increasingly less efficient as the letter is rotated away from the upright.

In order to perform mental rotation, the following information is generally thought to be available. First, prior to mental rotation, the letter is identified (Cooper \& Shepard, 1973; Corballis, 1988; Heil et al., 1996; Milivojevic et al., 2009), which allows for determining the orientation in which the stimulus is presented and the normal upright orientation allowing for the shortest direction for mental rotation to be determined. All that remains is to polarize the letter's horizontal axis. Mental rotation of the stimulus to the upright polarizes the horizontal axis into left and right, by comparison with the viewer-centered coordinate system.

However, it is here suggested that the information above may be used to polarize the horizontal axis without actually performing the rotation. Imagine the capital letter R, normal version, rotated counterclockwise to any orientation less than $180^{\circ}$. To bring it to the upright requires executing a clockwise rotation. The rotational vector of the top pole of the $\mathrm{R}$ indicates a movement direction from the upright vertical leg of the $\mathrm{R}$ toward the curve and diagonal leg of the $\mathrm{R}$, which corresponds to a left-to-right movement in upright terms. The bottom pole would likewise move right to left. Similarly, if the $\mathrm{R}$ were presented rotated clockwise, the required direction of mental rotation would be counterclockwise, reversing the direction, producing a right-to-left code for the top pole in upright terms. In other words, the pole of the horizontal axis toward which the top pole moves could be coded as "right" or "left" by preparing, but not actually performing, a clockwise or counterclockwise rotation. When the stimulus is rotated $180^{\circ}$, the vertical axis of the object is 
aligned with the environmental vertical, but with opposite polarity. This alignment of the vertical axes may allow for a random direction of rotation to be assigned. Moreover, since this alignment of the vertical axes also occurs for the upright, even upright presentations may have a random direction assigned, even though the magnitude of the required rotation would be zero, and so this nonrotation process could be operating at all orientations and would produce an orientation-independent RT function. This suggested process dovetails nicely with one of the original assumptions that was made when starting out on this investigation - namely, that the nonrotation process would produce a flat function with a mean RT equal to the mean of the upright condition.

Why, then, is mental rotation required at all, and why is it more necessary as the letters are presented at more extreme orientations? To provide a resolution to this issue, it is suggested that as the letter is rotated further from the upright, the polarity as defined by the rotational direction information above conflicts with the left/right codes that are produced by the viewer-centered coordinate system, meaning that when a normal version of $\mathrm{R}$ is rotated $180^{\circ}$, the curve is actually on the viewer's left, whereas for a normal upright $R$, the curve is on the viewer's right. Although preparation for rotation in either direction could polarize the axis to assign the curve to the right side, the viewercentered coordinate system is coding it as left, creating maximal interference or spatial conflict. On the basis of the analysis, the need to resolve this conflict between the proposed spatial codes appears to be in proportion to the strength of the conflict, which itself increases as the axes increase in their misalignment. Again, this dovetails nicely with another of the initial assumptions, which is that mental rotation would always be required for $180^{\circ}$ presentations. However, maximal interference does not necessarily lead to the conclusion that resolving the conflict must be performed $100 \%$ of the time, and so the proposal can only be considered as tentatively supporting this assumption.

Under the present model, mental rotation is employed to resolve the conflict between the rotation-direction-induced polarity codes and the spatial codes of the viewer-centered coordinate system. Mental rotation would achieve this resolution by bringing the two codes into alignment, and it may be that mental rotation is necessary to do this, and not a double-check optional procedure, if the two codes are effectively canceling the polarization of the horizontal axis. Similar spatial compatibility effects operating as a function of stimulus orientation have been suggested to be at play in other tasks (Light \& Hamm, 2008). It also may be that conflict between the letter parts for a mirror stimulus conflict with the spatial coding of the long-term normal, a conflict that is resolved by the proposed out-ofplane flip (Hamm et al., 2004).

In short, it may be that the direction of required rotation acts as the source of information that allows for the polarization of the horizontal axis without requiring mental rotation. It may be that the direction of rotation information is able to benefit alphanumeric stimuli because of the importance of left and right with respect to the longterm representations of those characters. This process may not aid left-/right-facing decisions involving other common objects, because the long-term representations for those items would, presumably, code their horizontal axis in "front/back" terms, rather than left/right terms, and the left-/right-facing task may force comparison with the viewer-centered reference frame. Interestingly, direction of rotation does appear to produce response compatibility effects in left-/right-facing judgments with common objects (Jolicœur, 1988) when the RT functions for the separate responses are examined.

Finally, it appears that although clockwise and counterclockwise directions may be able to code for the left and right poles of the horizontal axis, these directions of the horizontal axis do not appear to code the vertical axis into top and bottom. This is indicated by the finding above that stimuli with symmetry about the horizontal axis, such as b and p (Corballis \& McLaren, 1984), appear to consistently require mental rotation in order to polarize the vertical axis. This suggests that rotational direction has a greater correspondence to horizontal directions than to vertical directions.

In summary, it appears that the RTs for rotated mirror/ normal letter discriminations can be modeled by the combination of three components. The first component is the mean RT on those trials that require mental rotation, which produces a linear increase in $\mathrm{RT}$ as a function of the orientation of the stimulus. The proportion of trials that require mental rotation appears to be in direct proportion to the degree to which the stimulus is inverted. The second component is a nonrotation method of response determination, suggested to be based on the codes applied to the horizontal axis by determining whether either a clockwise or a counterclockwise transformation is required. What influences the mixture ratio between these two processing routes, what manipulations may benefit or penalize the nonrotation process, and whether these two processes operate serially or in parallel remain to be determined. The third and final component to the RT is the mirror-normal difference, which appears to be related to an individual's mental rotation rate. This relationship is consistent with the notion that this difference reflects an out-of-plane flip of the stimulus item (Hamm et al., 2004).

In closing, it should be noted that the possibility of a second orientation-sensitive process other than mental rotation being involved in rotated mirror/normal letter discriminations has obvious implications for the interpretation of orientation effects found in brain-imaging data. Which linear effect is being measured? Do the linear effects of orientation found during brain imaging reflect the prolonged engagement of mental rotation as a process, the proportion of times that mental rotation is employed, or perhaps the increase in conflict between incompatible spatial codes that leads to the increased failure rate of the proposed nonrotation route?

\section{AUTHOR NOTE}

This research was conducted in partial fulfillment of the requirements for the degree of BSc (Hons), awarded to the first author. We thank four anonymous reviewers for their comments and suggestions on a previous version of the manuscript. Correspondence concerning 
this article should be addressed J. P. Hamm, Research Centre for Cognitive Neuroscience, Department of Psychology, University of Auckland, City Campus, Private Bag 92019, Auckland, New Zealand 1142 (e-mail: j.hamm@auckland.ac.nz).

\section{REFERENCES}

Cooper, L. A., \& Shepard, R. N. (1973). Chronometric studies of the rotation of mental images. In W. G. Chase (Ed.), Visual information processing (pp. 75-176). New York: Academic Press.

Corballis, M. C. (1988). Recognition of disoriented shapes. Psychological Review, 95, 115-123.

Corballis, M. C., \& McLaren, R. (1984). Winding one's Ps and Qs: Mental rotation and mirror-image discrimination. Journal of Experimental Psychology: Human Perception \& Performance, 10, 318-327.

Corballis, M. C., \& McMaster, H. (1996). The roles of stimulusresponse compatibility and mental rotation in mirror-image and leftright decisions. Canadian Journal of Experimental Psychology, 50, 397-401.

Corballis, M. C., \& Nagourney, B. A. (1978). Latency to categorize disoriented alphanumeric characters as letters or digits. Canadian Journal of Psychology, 32, 186-188.

Corballis, M. C., Zbrodoff, N. J., Shetzer, L. I., \& Butler, P. B. (1978). Decisions about identity and orientation of rotated letters and digits. Memory \& Cognition, 6, 98-107.

Duncombe, M. E., Bradshaw, J. L., Iansek, R., \& Phillips, J. G. (1994). Parkinsonian patients without dementia or depression do not suffer from bradyphrenia as indexed by performance in mental rotation tasks with and without advance information. Neuropsychologia, 32, 1383-1396.

Haмm, J. P. (2001). Object-oriented millisecond timers for the PC. Behavior Research Methods, Instruments, \& Computers, 33, 532-539.

Hamm, J. P., Johnson, B. W., \& Corballis, M. C. (2004). One good turn deserves another: An event-related brain potential study of rotated mirror-normal letter discriminations. Neuropsychologia, 42, 810-820.

HeAthCote, A. (1988). Screen control and timing routines for the IBM microcomputer family using a high-level language. Behavior Research Methods, Instruments, \& Computers, 20, 289-297.

Heil, M., Bajric, J., Rosler, F., \& Hennighausen, E. (1996). Eventrelated potentials during mental rotation: Disentangling the contributions of character classification and image transformation. Journal of Psychophysiology, 10, 326-335.
Heil, M., Rauch, M., \& Hennighausen, E. (1998). Response preparation begins before mental rotation is finished: Evidence from eventrelated brain potentials. Acta Psychologica, 99, 217-232.

JoLICEUR, P. (1988). Mental rotation and the identification of disoriented objects. Canadian Journal of Psychology, 42, 239-243.

Joliceur, P. (1990). Identification of disoriented objects: A dualsystems theory. Mind \& Language, 5, 387-410.

Light, P., \& HAMM, J. P. (2008). Stimulus-response compatibilities during top-bottom discriminations. Canadian Journal of Experimental Psychology, 62, 81-90.

McClelland, J. L. (1979). On the time relations of mental processes: An examination of systems of processes in cascade. Psychological Review, 86, 287-330.

Milivojevic, B., Hamm, J. P., \& Corballis, M. C. (2009). Functional neuroanatomy of mental rotation. Journal of Cognitive Neuroscience, 21, 945-959.

MurRaY, J. E. (1997). Flipping and spinning: Spatial transformation procedures in the identification of rotated natural objects. Memory \& Cognition, 25, 96-105.

OLDFIELD, R. C. (1971). The assessment and analysis of handedness: The Edinburgh inventory. Neuropsychologia, 9, 97-113.

Shepard, R. N., \& Metzler, J. (1971). Mental rotation of threedimensional objects. Science, 171, 701-703.

VAn Selst, M., \& Jolicceur, P. (1994). A solution to the effect of sample size on outlier elimination. Quarterly Journal of Experimental Psychology, 3A, 631-650.

\section{NOTES}

1. The proposed calculation of proportions takes advantage of the property of the arithmetic mean that if one combines the scores from two distributions, the arithmetic mean of the combined distribution will be the weighted means of the two individual distributions, regardless of the shape of those distributions. This does not apply to other measures of central tendency, such as the median, the geometric mean ( $n$th root of the product of $n$ scores), or the harmonic mean (the reciprocal of the mean of the reciprocal of the scores).

2. Note that the curved function is found in all blocks, including the first, and so does not appear to be a result of practice. Moreover, the model proposed produces a good fit for all individual blocks, suggesting there was no change in the proportions that make up the mixture over the course of the experiment. 
APPENDIX

Table A1

Fits for Linear and Mixture Models

\begin{tabular}{crrrrrr} 
& & \multicolumn{3}{c}{ SS } & & \multicolumn{2}{c}{$\%$ Reduction } \\
\cline { 3 - 4 } \cline { 6 - 7 } Participant & & \multicolumn{1}{c}{ Linear } & Mixture & & Linear & Mixture \\
Number & SS Mean & \multicolumn{1}{c}{ Model } & Model & & Model & Model \\
\hline 1 & 369,181 & 133,889 & $\mathbf{4 1 , 2 8 6}$ & & 64 & $\mathbf{8 9}$ \\
2 & 240,699 & $\mathbf{3 1 , 3 3 2}$ & 61,117 & & $\mathbf{8 7}$ & 75 \\
3 & 335,656 & 218,330 & $\mathbf{5 5 , 1 3 4}$ & & 35 & $\mathbf{8 4}$ \\
4 & 200,125 & 149,487 & $\mathbf{4 0 , 2 6 0}$ & & 25 & $\mathbf{8 0}$ \\
5 & 636,355 & 366,096 & $\mathbf{1 0 2 , 3 4 4}$ & & 42 & $\mathbf{8 4}$ \\
6 & 374,075 & 164,690 & $\mathbf{9 7 , 5 8 0}$ & & 56 & $\mathbf{7 4}$ \\
7 & 233,633 & 42,444 & $\mathbf{1 8 , 1 3 2}$ & & 82 & $\mathbf{9 2}$ \\
8 & 864,194 & $\mathbf{6 9 , 5 0 3}$ & 158,137 & & $\mathbf{9 2}$ & 82 \\
9 & 767,322 & 286,623 & $\mathbf{1 3 8 , 2 2 3}$ & & 63 & $\mathbf{8 2}$ \\
10 & $1,048,030$ & $\mathbf{8 0 , 3 9 8}$ & 239,197 & & $\mathbf{9 2}$ & 77 \\
11 & 448,419 & 153,073 & $\mathbf{5 4 , 8 8 1}$ & & 66 & $\mathbf{8 8}$ \\
12 & 788,663 & 894,822 & $\mathbf{2 9 6 , 6 6 0}$ & & -13 & $\mathbf{6 2}$ \\
13 & 149,867 & 145,795 & $\mathbf{6 1 , 2 5 1}$ & & 3 & $\mathbf{5 9}$ \\
14 & $1,922,072$ & $1,050,104$ & $\mathbf{2 6 1 , 1 1 0}$ & & 45 & $\mathbf{8 6}$ \\
15 & 326,021 & 132,082 & $\mathbf{5 8 , 6 1 9}$ & & 59 & $\mathbf{8 2}$ \\
16 & 526,024 & 265,846 & $\mathbf{8 3 , 0 0 8}$ & & 49 & $\mathbf{8 4}$ \\
17 & 568,681 & 170,937 & $\mathbf{4 8 , 2 3 0}$ & & 70 & $\mathbf{9 2}$ \\
18 & $1,794,759$ & 772,015 & $\mathbf{4 8 4 , 0 3 9}$ & & 57 & $\mathbf{7 3}$ \\
19 & $1,085,594$ & 320,164 & $\mathbf{1 1 7 , 6 9 6}$ & & 71 & $\mathbf{8 9}$ \\
20 & 640,646 & $\mathbf{1 4 3 , 8 5 2}$ & 155,053 & & $\mathbf{7 8}$ & 76 \\
21 & 456,833 & 219,899 & $\mathbf{3 5 , 7 9 7}$ & & 52 & $\mathbf{9 2}$ \\
22 & 296,689 & 191,157 & $\mathbf{4 2 , 8 8 3}$ & & 36 & $\mathbf{8 6}$ \\
23 & 168,239 & 53,783 & $\mathbf{3 7 , 0 6 6}$ & & 68 & $\mathbf{7 8}$ \\
24 & 602,303 & 539,486 & $\mathbf{1 7 4 , 3 5 3}$ & & 10 & $\mathbf{7 1}$ \\
\hline
\end{tabular}

Note-The sum of squared deviations (SSs) from a participant's grand mean (column 2), from a model that fits only a linear estimation of the orientation effect (column 3), and from the mixture model (column 4). Columns 5 and 6 present the models' effectiveness at predicting individual participant performance as a percentage of the reduction of the deviation about the mean, with boldface values highlighting the better fit. The italicized negative value for Participant 12 highlights the fact that the models could potentially increase the variance relative to the mean (a negative improvement). Both the linear and mixture models estimate the mirror normal difference on the basis of rotation rates and do not model the interaction. Participants 1-12 are male; 13-24 are female.

The following figures show the observed mean RTs in milliseconds for normal (filled circles) and mirrored (open squares) trials for all the participants as a function of the orientation of the letter in degrees clockwise from the upright $\left(30^{\circ}\right.$ steps $)$. Solid lines show the model predictions for normal trials, and dashed lines show the model predictions for mirrored trials. The presented model curves do not model the observed facing $\times$ orientation interaction but use a common rate of rotation for both mirror and normal predictions for the proportion of trials thought to require mental rotation. The separation between mirror and normal trials is also estimated from this rate of rotation. 

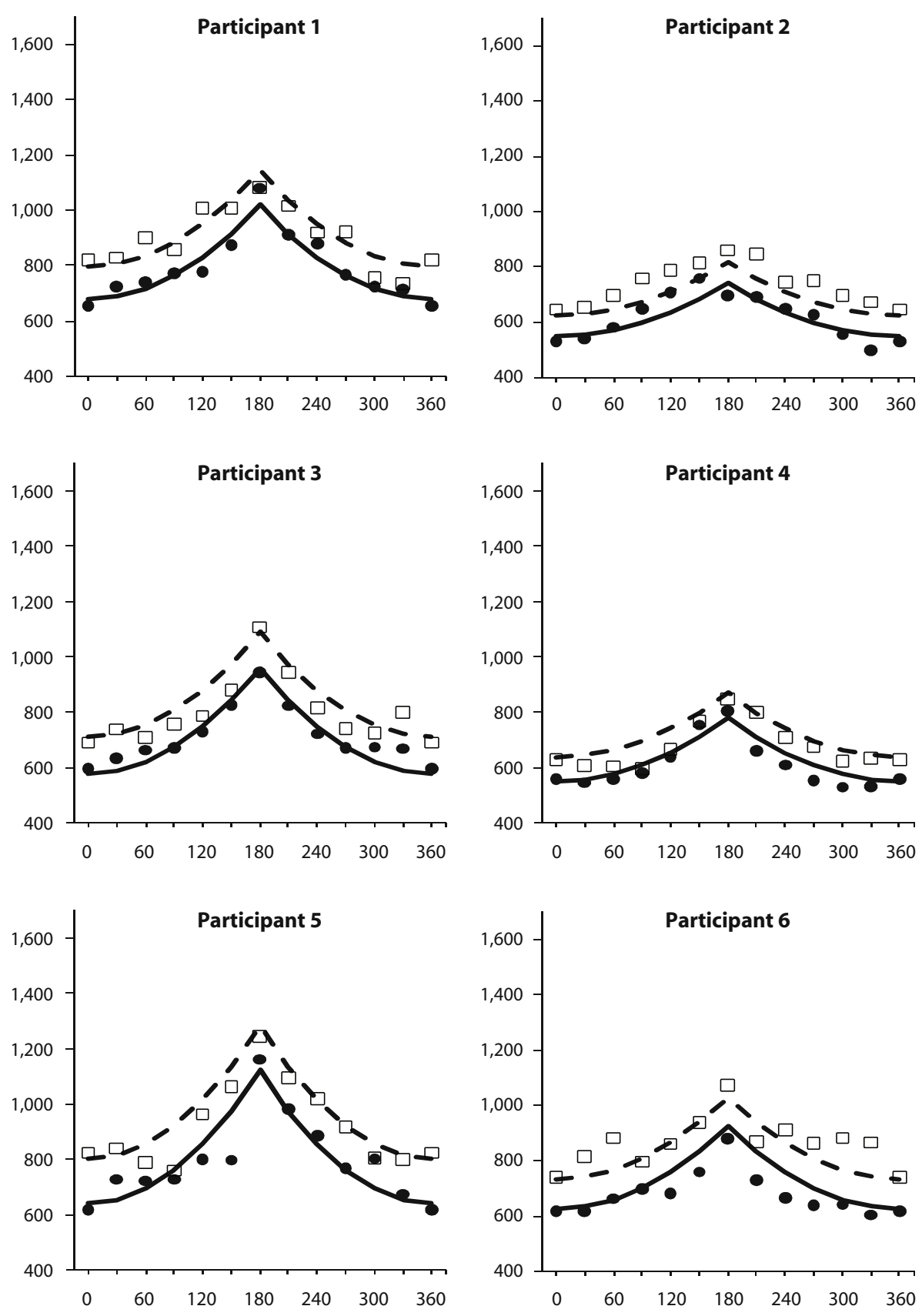

$$
\begin{aligned}
& \text { - Data normal } \quad \text { Mixed normal } \\
& \square \text { Data mirror } \quad \text { Mixed mirror }
\end{aligned}
$$



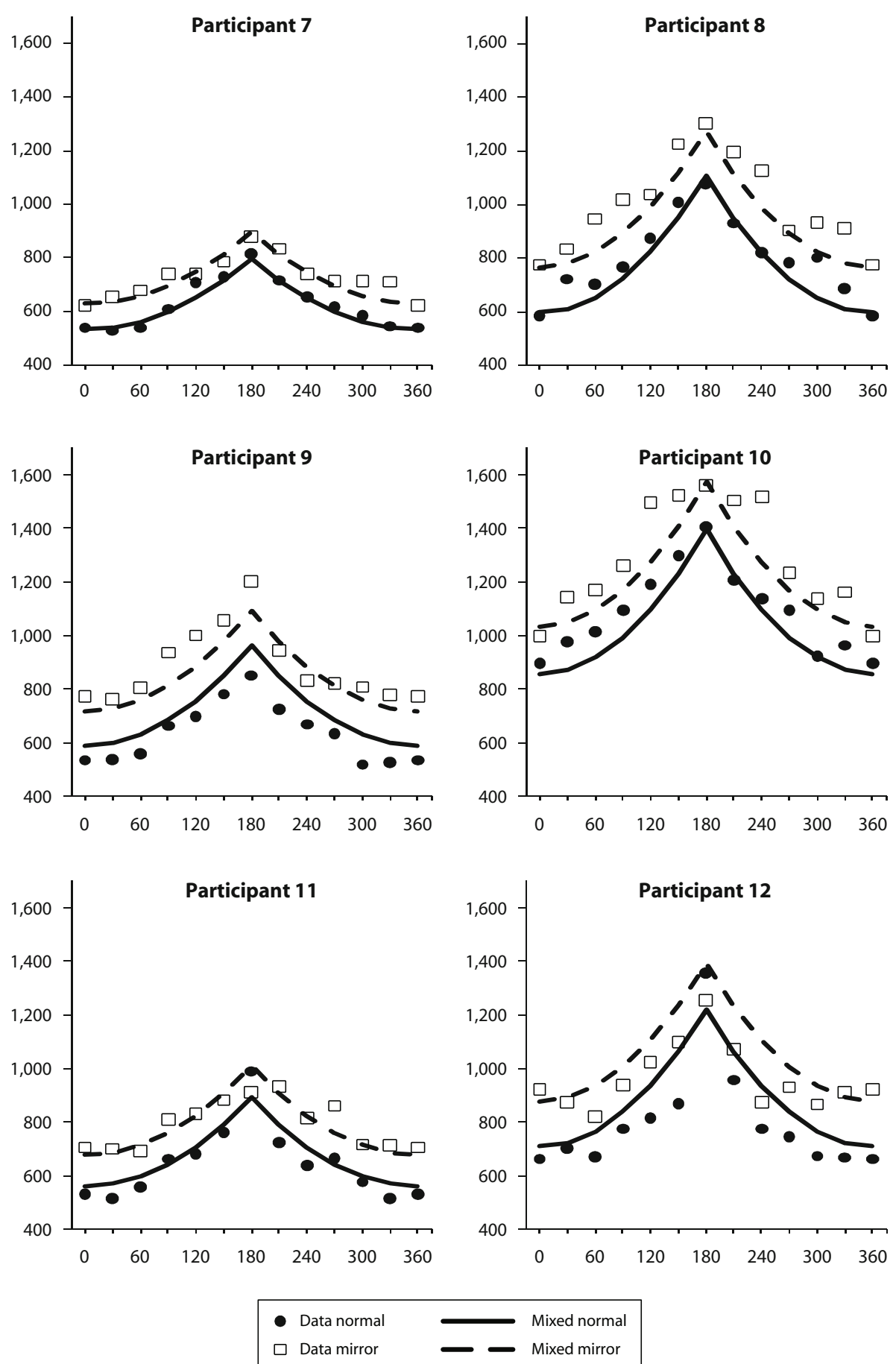

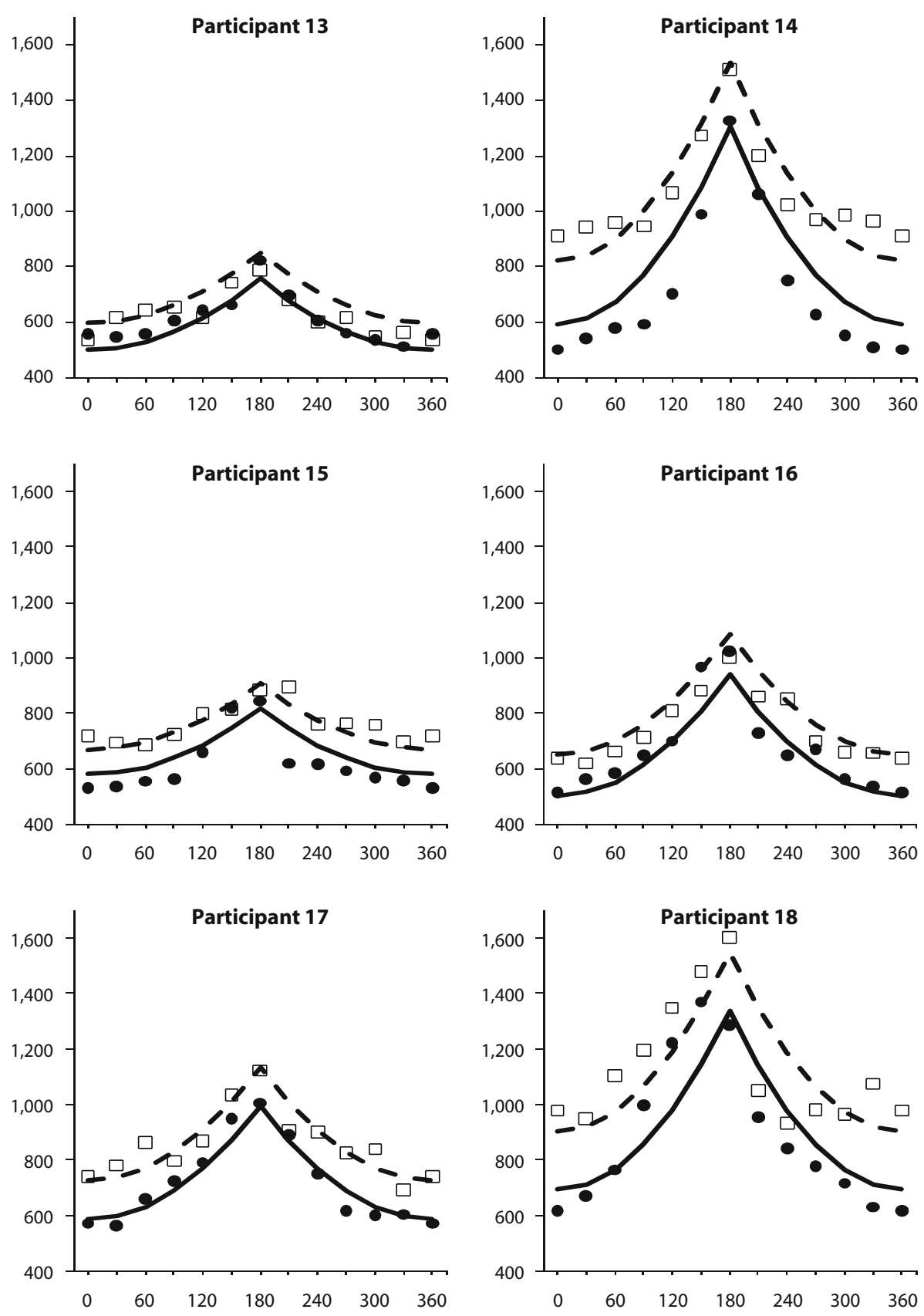

$$
\begin{aligned}
& \text { - Data normal } \quad \text { Mixed normal } \\
& \square \text { Data mirror } \quad \text { Mixed mirror }
\end{aligned}
$$



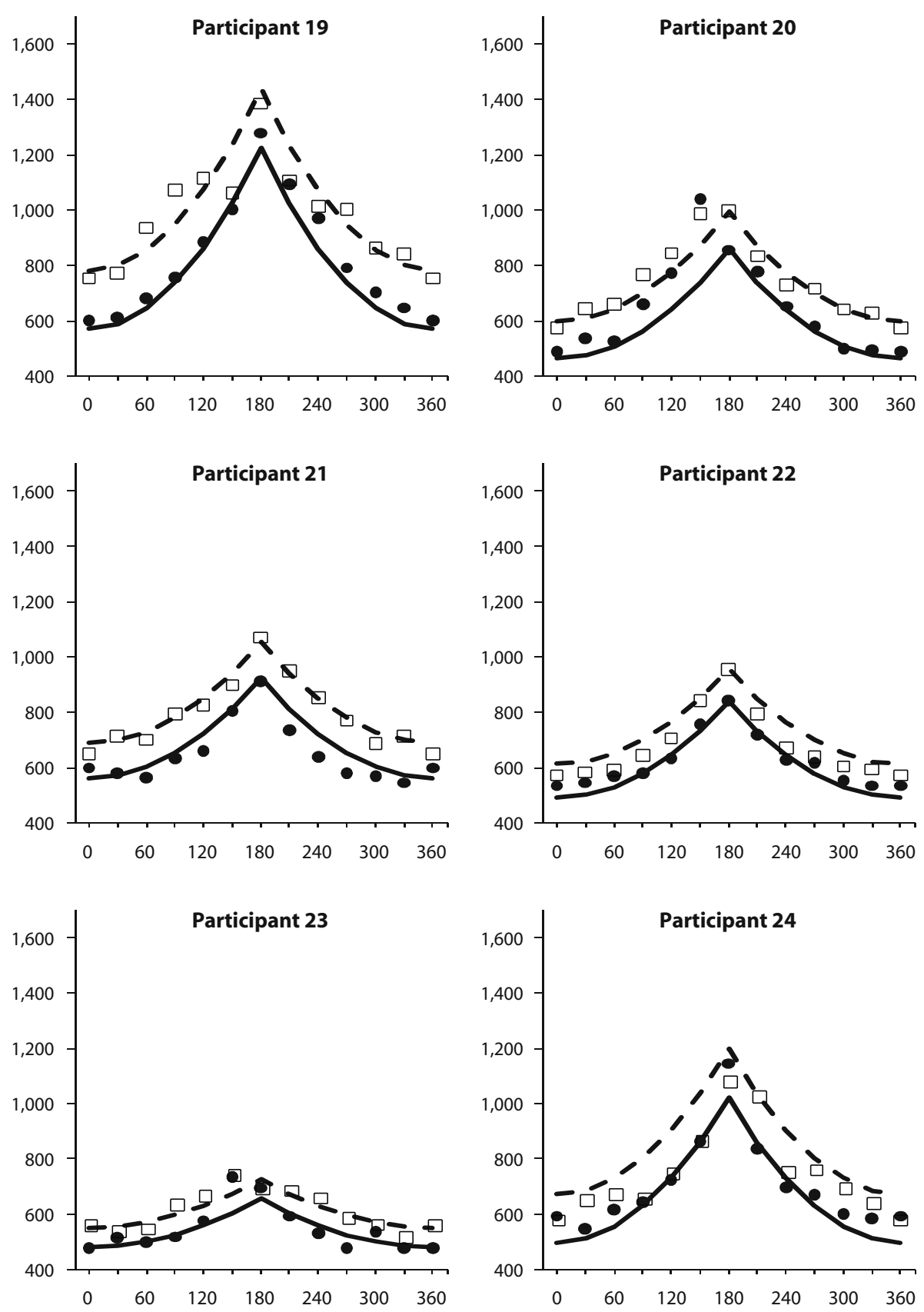

$$
\begin{aligned}
& \text { Data normal } \quad \longrightarrow \text { Mixed normal } \\
& \square \text { Data mirror } \quad-\text { Mixed mirror }
\end{aligned}
$$

